\title{
Adaptive fuzzy internal model control of thermal heating process
}

\author{
Muhammad Shafiq ${ }^{\mathrm{a})}$ and Agus R Widodo ${ }^{\mathrm{b})}$ \\ Systems Engineering Department, King Fahd University of Petroleum \& Minerals \\ PO Box 1381, Dhahran 31261, Saudi Arabia \\ a)mshafiq@ccse.kfupm.edu.sa \\ b)arwidodo@ccse.kfupm.edu.sa
}

Abstract: An adaptive fuzzy internal model control (IMC) scheme is proposed to control nonlinear systems with inherent time delay. A Takagi-Sugeno (TS) fuzzy modeling with adaptive tuner is used to obtain the plant model. TS model is obtained using experimental data of step responses at different input levels and the model accuracy is improved using the normalized least mean squares (nLMS) based online adaptive tuner. Adaptive inverse of the plant is established using adaptive inverse control scheme on TS model with adaptive tuner. The scheme is implemented on a laboratory scale heating process. The experimental results indicate that the heating process output tracks the reference signal.

Keywords: internal model control, adaptive inverse control, TakagiSugeno fuzzy modeling, adaptive tuner

Classification: Science and engineering for electronics

\section{References}

[1] Feedback®, Process Control Workshop 37-001, http://www.fbk.com

[2] M. Morari and E. Zaferiou, Robust Process Control, Prentice Hall, NJ, 1989.

[3] T. Takagi and M. Sugeno, "Fuzzy identification of systems and its application to modeling and control," IEEE Trans. Syst., Man, Cybern., vol. 15, no. 1, pp. 116-132, 1985.

[4] W. F. Xie and A. B. Rad, "Fuzzy Adaptive Internal Model Control," IEEE Trans. Ind. Electron., vol. 47, no. 1, pp. 192-202, 2000.

[5] A. Fink, O. Nelles, and R. Isermann, "Nonlinear internal model control for MISO systems based on local linear neuro-fuzzy models," 15th IFAC World Congress, Barcelona, 2002.

[6] B. Widrow and E. Walach, Adaptive Inverse Control, Prentice Hall, NJ, 1996.

[7] M. Shafiq and S. H. Riyaz, "Internal model control structure using adaptive inverse control strategy," IEEE Conf. Control and Automation, 2003. 


\section{Introduction}

A thermal heating process typically consists of a blower, a heating grid, tube, and a temperature sensor (see Fig. 1). A variable power supply, $P(t)$, provides power to the heater. The air temperature at some desired location in the process tube, $T(t-\tau)$, provides the process output. In order to make the heating process follow a desired pattern, a real-time computer controlled mechanism can be implemented by initiating an appropriate controlling signal from the computer to affect the heater power and by observing the temperature output [1].

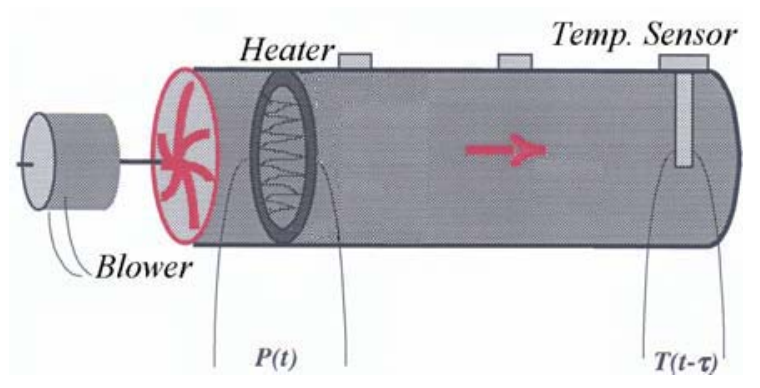

Fig. 1. Thermal heating process.

Among various process control strategies, internal model control (IMC) offers a number of advantages. Composed of the explicit model of the plant and a stable feed-forward (plant inverse) controller, IMC design is simple and straightforward. A "perfect" control is theoretically achievable if the model exactly resembles the actual plant. The IMC controller guarantees the internal stability of the closed-loop system and the controller's parameters can be tuned online easily without disturbing stability of the system [2].

In real life the exact model of the plant may not be known. Most often plants are nonlinear and their parameters may be time varying. In this case the implementation of IMC is not straight forward. In IMC inverse of the plant is required to accomplish the tracking objective. The precision of the plant inverse depends on the model accuracy. We propose to obtain the model of the plant based on Takagi-Sugeno model with adaptive model tuner. This adaptive tuner is composed of adaptive finite impulse response (FIR) filter, which is inherently stable. Adaptive model tuner is added-in to enhance model accuracy. Stable inverse of the model is obtained by adaptive inverse control strategy.

\section{Takagi-Sugeno Fuzzy Modeling}

The major requirement of designing IMC is an exact model of the plant. Among the existing modeling approaches, Takagi-Sugeno fuzzy modeling provides an effective way to represent nonlinear systems by combining a rulebased description with local functional description. The fuzzy model of a system is built on a fuzzy partition of input space. In each fuzzy subspace a local linear input-output relation is formed. The output of fuzzy reasoning is 
given by the aggregation of the values inferred by some implications applied to an input [3].

The characteristic of the nonlinear system is approximated by a group of fuzzy rules shown as follows:

$$
R_{i} \text { if } x_{i, 1}(t) \text { is } A_{i, 1} \ldots x_{i, N} \text { is } A_{i, N} \text {, then } \hat{y}_{i}(s)=H_{i}(s) u(s)
$$

Where $x_{i, j}(t)$ is input space variable, such as plant input $u(t)$, plant output $y(t)$, modeling error $e(t)$, etc.; $A_{i, j}$ represents linguistic constant; $i=1: M$ (the number of the fuzzy rules); $j=1: N$ (the number of input space); $\hat{y}_{i}(t)$ is the output of the $i^{\text {th }}$ local linear system, and $H_{i}(s)$ is the rational transfer function of the local linear system.

Let local transfer function be given as

$$
H_{j}(s)=\frac{\hat{y}_{j}(s)}{u(s)}=\frac{b_{m}^{j} s^{m-1}+b_{m-1}^{j} s^{m-2}+\ldots b_{1}^{j}}{s^{n}+a_{n}^{j} s^{n-1}+a_{n-1}^{j} s^{n-2}+\ldots a_{1}^{j}}
$$

where the parameters $a_{1}, \ldots, a_{n}$ and $b_{1}, \ldots, b_{m}$ have to be identified. The identification of the parameters can be done by using different strategies like recursive least squares (RLS), artificial neural networks, step response analysis and etc $[4,5]$.

The final output $y(t)$ of the nonlinear system, which is inferred from the implication of the fuzzy rules, is given as the weighted average of all $\hat{y}_{i}(t)$.

$$
\hat{y}(t)=\frac{\sum_{i=1}^{M} \hat{y}_{i}(t) \beta\left(\hat{y}_{i}(t)\right)}{\sum_{i=1}^{M} \beta\left(\hat{y}_{i}(T)\right)}
$$

where

$$
\beta\left(\hat{y}_{i}(t)\right)=\beta_{A_{i, 1}}\left(x_{i, 1}(t)\right) \beta_{A_{i, 2}}\left(x_{i, 2}(t)\right) \ldots \beta_{A_{i, N}}\left(x_{i, N}(t)\right)
$$

is the truth value of the consequence of each fuzzy rule; $\beta_{A_{i, j}}\left(x_{i, j}(t)\right)$ is the fuzzy membership function of the fuzzy set $A_{i, j}$.

In the proposed adaptive IMC scheme (see Fig. 2), fuzzy adaptive model of the plant is obtained as a combination of TS fuzzy model and adaptive FIR filter. Local linear models of the nonlinear plant are obtained offline from

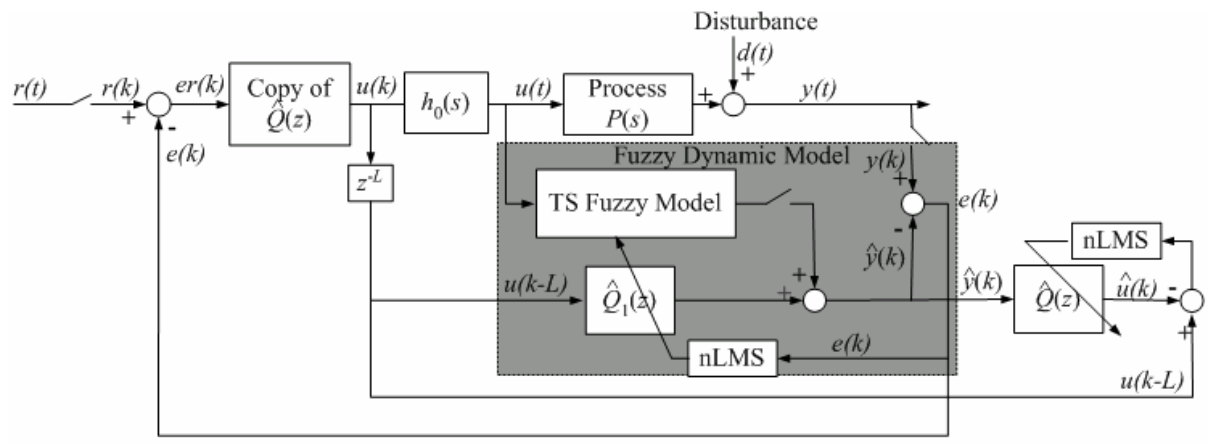

Fig. 2. AIC based adaptive fuzzy IMC configuration. 
the step responses at different input levels and separate transfer functions are obtained at each input level. Then, the Takagi-Sugeno fuzzy model is constructed to fuzzily switch among the transfer functions depending upon the input level. An adaptive FIR filter based adaptive tuner $Q_{1}(z)$ is added in parallel to the fuzzy model to obtain a fuzzy adaptive model of the plant. The parameters of $Q_{1}(z)$ are updated by nLMS.

\section{Adaptive Controller Design}

Consider a continuous-time, stable-plant transfer function $P(s)$. Let $h_{0}(s)$ denote the zero-order hold. The discrete-time version of the plant is $P(z)$. $u(k)$ is the control input to the plant and $y(k)$ is output of the plant. The control objective is to synthesize $u(k)$ such that $y(k)$ tracks some bounded piecewise continuous desired trajectory $r(k)$. The inverse of plant model, $Q(z)$, can be incorporated in the feed-forward path to achieve asymptotic tracking in IMC structure. In this scheme the inverse of plant model is estimated as an adaptive FIR filter using nLMS algorithm [6]. This approximate inverse system is the local inverse of the system. The parameters of this inverse may change with variation in the system excitation signal [7].

The method to estimate the approximate inverse is briefly described below. Let $\hat{u}(k)$ be the predicted controller output, required to track $u(k-L)$, a $L$-sample delayed $u(k)$. Then

$$
\hat{u}(k)=W^{T}(k) \varphi(k)
$$

where $W(k)$ is an array consisting of filter parameters and $\varphi^{T}(k)=[\hat{y}(k)$, $\hat{y}(k-1), \ldots, \hat{y}(k-p)]$.

The parameter adjustment law for the approximate inverse filter based on nLMS is given by

$$
W(k)=W(k-1)+\mu \frac{\varphi(k)}{\nu+\varphi(k)^{T} \varphi(k)} \varepsilon(k)
$$

where $\varepsilon(k)=u(k-L)-\hat{u}(k)$ and a small constant $\nu$ is introduced in the estimator to avoid division by zero when $\varphi(k)^{T} \varphi(k)$ is very small and $0<$ $\mu<1$ is the learning rate.

\section{Experimental Results}

The proposed scheme is implemented on laboratory scale temperature control of a heating process. Standard IBM PC-type Pentium III is used for the computation in real time. Data acquisition is accomplished by Advantech card PCI-1711 and the controller is implemented in Simulink real-time windows target environment. The computations are performed in floating-point format and the sampling interval for the temperature of the heating system is selected 0.1 seconds.

Experimental work on the process step responses at 7 different input levels produces local transfer functions valid for each respective input level. Since 
the plant input voltage $u(t)$ is the only manipulating variable, therefore only one fuzzy input space exists. Thus, the TS fuzzy rule is as follows:

$$
R_{i} \text { if } u(t) \text { is } A_{i} \text { then } \hat{y}_{i}(s)=H_{i}(s) u(s) .
$$

Triangular and trapezoidal membership functions are used to produce the truth value of the logical variables $A_{i}$ 's (see Fig. 3 (a)).

Figure 3 (b) shows that output (temperature) of the process converges to the desired temperature quickly. It can be confirmed from Fig. 3(c) that control input to the plant remains bounded. Plant parameters are shown in Fig. $3(d)$.

\section{Conclusion}

In this paper, an adaptive fuzzy internal model control scheme has been proposed to control a nonlinear process. Takagi-Sugeno fuzzy model with adaptive tuner has been used to obtain the process internal model. The plant inverse has been obtained through adaptive FIR filters using normalized least mean squares algorithm. The experimental results have demonstrated the effectiveness of the IMC scheme in tracking problem.

\section{Acknowledgments}

The authors acknowledge King Fahd University of Petroleum and Minerals, Dhahran 31261 Saudi Arabia, for supporting this research. 


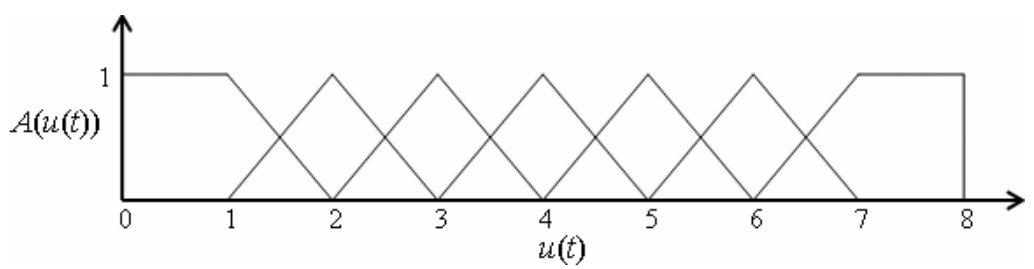

(a)

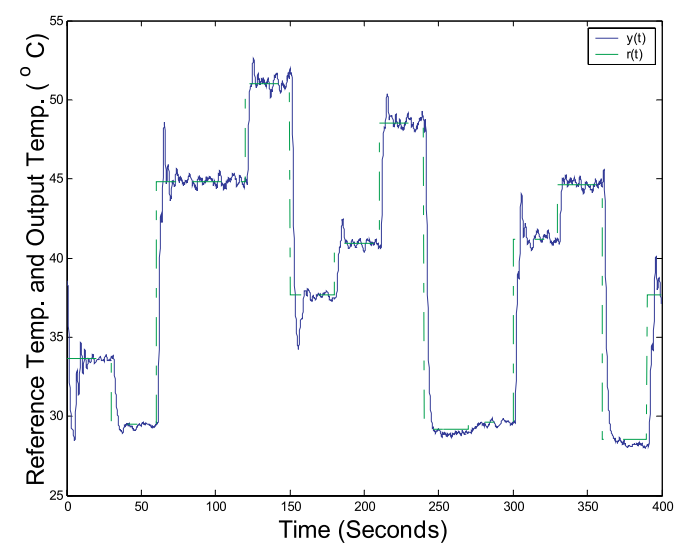

(b)

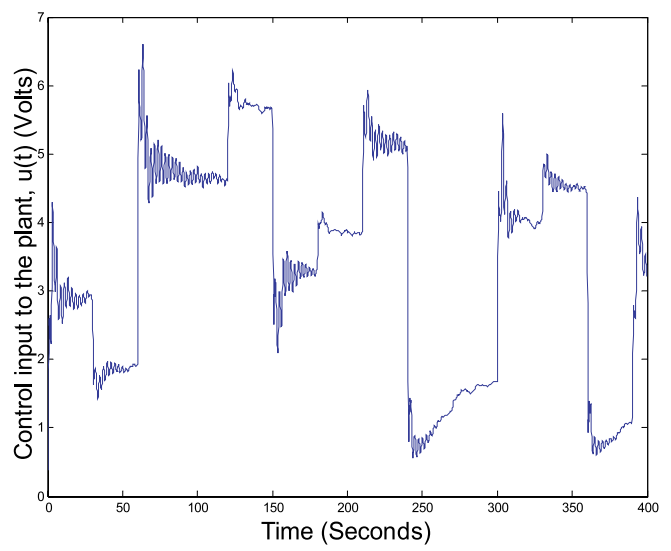

(c)

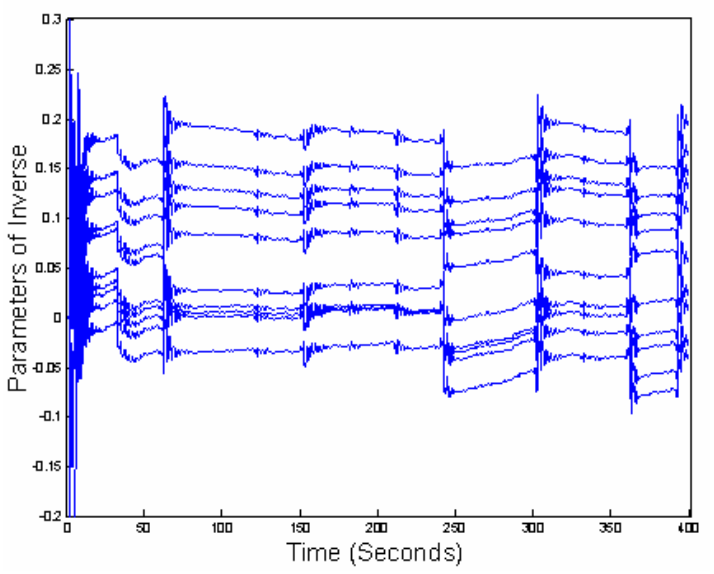

(d) (c) IEICE 2004

DOI: 10.1587/elex.1.120

Received April 26, 2004

Accepted May 07, 2004

Published June 25, 2004 